\title{
Phosphate Metabolism in CKD Stages 3-5: Dietary and Pharmacological Control
}

\begin{abstract}
Markus Ketteler
Division of Nephrology, Klinikum Coburg, 96450 Coburg, Germany

Correspondence should be addressed to Markus Ketteler, markus.ketteler@klinikum-coburg.de

Received 21 December 2010; Accepted 14 March 2011

Academic Editor: Domenico Russo

Copyright () 2011 Markus Ketteler. This is an open access article distributed under the Creative Commons Attribution License, which permits unrestricted use, distribution, and reproduction in any medium, provided the original work is properly cited.

When compared to the available information for patients on dialysis (CKD stage 5D), data on the epidemiology and appropriate treatment of calcium and phosphate metabolism in the predialysis stages of chronic kidney disease (CKD) are quite limited. Perceptible derangements of calcium and phosphate levels start to become apparent when GFR falls below $30 \mathrm{~mL} / \mathrm{min}$ in some, but not all, patients. However, hyperphosphatemia may be a significant morbidity and mortality risk predictor in predialysis CKD stages. The RIND study, evaluating progression of coronary artery calcification in incident hemodialysis patients, indirectly demonstrated that vascular calcification processes start to manifest in CKD patients prior to the dialysis stage, which may be closely linked to early and invisible derangements in calcium and phosphate homeostasis. Novel insights into the pathophysiology of calcium and phosphate handling such as the discovery of FGF23 and other phosphatonins suggest that a more complex assessment of phosphate balance is warranted, possibly including measurements of fractional phosphate excretion and phosphatonin levels in order to appropriately evaluate disordered metabolism in earlier stages of kidney disease. As a consequence, early and preventive treatment approaches may have to be developed for patients in CKD stages 3-5 to halt progression of CKD-MBD.
\end{abstract}

\section{Introduction}

Availability of phosphate is of key importance for a number of normal physiological body functions including bone development, cellular membrane integrity (phospholipid content), and energy transfer (mitochondrial metabolism). Normal phosphate homeostasis, thus, maintains serum concentrations quite constantly between 0.8 and $1.45 \mathrm{mmol} / \mathrm{L}$ in the normal population. In this line, meals containing a high phosphate load in most instances do not acutely raise the serum phosphate levels, because phosphaturia may become augmented by as yet undefined mechanisms [1]. Furthermore, serum phosphate levels show rather a circadian rhythm which does not parallel intake (phosphate peaks in the early morning hours) [2]. In health, the kidney excretes approximately two thirds of the daily dietary phosphate load, and even with deteriorating kidney function in CKD, this excretion rate remains relatively well maintained [3]. Acute "unphysiological" oral phosphate intake is probably managed by the so-called phosphatonins or by adaptations of the filtered load $[3,4]$. It appears that the body activates a number of potent systems in order to avoid both the occurrence of hyperphosphatemia.

\section{Epidemiology of Hyperphosphatemia in CKD Patients}

Kestenbaum and colleagues were the first to publish data from a large observational study studying the relationship between phosphate serum levels and outcomes in a CKD cohort [5]. More than 6,000 CKD patients were crosssectionally available for analysis, while 3,490 of them had a serum phosphate measurement during the previous eighteen months. The major result was that phosphate serum levels were found to be in the normal range until a GFR of $30 \mathrm{~mL} / \mathrm{min}$ was reached; only from then on average phosphate levels started to rise beyond the upper normal range and started to become inversely related to GFR, while it is yet unclear when serum phosphate levels tend to rise "within" the normal range in CKD [6]. In Kestenbaum's study, serum phosphate levels above $3.5 \mathrm{mg} / \mathrm{dL}$ were already 
significantly associated with mortality. Further, mortality risk progressively increased with each $0.5-\mathrm{mg} / \mathrm{dL}$ increase in phosphate serum levels. In a prospective, community-based, noninterventional, prospective cohort study (SEEK study, $n=1814$ CKD patients), Levin and colleagues were able to demonstrate similar findings with regard to the incidence of "visible" hyperphosphatemia-phosphate serum levels rose perceptibly in subjects in CKD stage 4 [7]. Therefore, both studies support the assumption that hyperphosphatemia may just be the final indicator (tip of an iceberg) of an already previously failing phosphate balance.

\section{Phosphate Homeostasis in CKD}

Up to $80 \%$ of the estimated daily phosphate intake of $20 \mathrm{mg} / \mathrm{kg}$ b.w. per day is intestinally absorbed in healthy individuals and circulates into an "extracellular phosphate pool" [3]. $3 \mathrm{mg} / \mathrm{kg}$ b.w. phosphate per day are exchanged between this virtual pool and the bone, if bone turnover is physiologically balanced. Two-thirds of the daily intake is excreted via healthy kidneys, the other third is excreted by the faeces resulting in a neutral phosphate balance. Kidney disease interferes with the ability to excrete phosphate loads; however, excretion remains surprisingly stable until the late stages of CKD.

The fact that there is a delayed onset of hyperphosphatemic episodes in CKD is probably based on counterregulatory actions of FGF23 and PTH [8-11]. Both hormones are causing phosphaturia when elevated and apparently become stimulated as early as in CKD stage 3 , connected to vitamin D status and dietary phosphate intake. Both in animal models as well as in human studies, it was shown that increases in serum FGF23 levels were paralleled by rises in renal fractional (relative) phosphate excretion counteracting increases in phosphate retention and subsequent hyperphosphatemia [811]. It is currently considered that a positive phosphate balance triggers an osteocyte "phosphate sensor" towards signalling release of FGF23. In this context, it is yet unknown, whether phosphate itself is the circulating messenger, or whether for example intestinal phosphatonins may serve as FGF23 inducers [3]. Furthermore, FGF23 and PTH secretion may be linked to each other. FGF23 actively suppresses calcitriol synthesis, thus antagonizing a central PTHsuppressive mechanism $[9,10]$. Therefore, the organism employs two different hormonal systems in order to shelter the body from phosphate accumulation, that is, hyperphosphatemia. There are also data that PTH and FGF23 secretion are directly interconnected, that is, that FGF23 inhibits PTH release via stimulating the dimeric klotho/FGF-receptor at the parathyroid gland [12]. PTH may vice versa inhibit FGF23 release from the osteocyte [12].

With this in mind, the question arises how acute dietary phosphate ingestion may influence phosphate, FGF23, and $\mathrm{PTH}$ regulation in CKD? Isakova et al. studied a group of CKD patients (GFR $20-45 \mathrm{ml} / \mathrm{min}$ ) versus healthy individuals ingesting a meal with a phosphate content of $500 \mathrm{mg}$ of phosphate [1]. Within 4 hours following ingestion, they found no increase in serum phosphate levels in both groups, but a rapid augmentation of fractional phosphate excretion in the healthy control group, while the baseline level of urinary excretion was already twice as high in the CKD cohort, with a nonsignificant trend towards a further increase. This phosphaturic response was not acutely accompanied by increases in FGF23 or PTH levels, while at baseline these two parameters were also already significantly elevated in the CKD group versus the healthy controls. This study had a few limitations including the fact that the exact timing of interventions was not presented. Nevertheless, this response excludes FGF23 as the predominant "acute-phase" phosphatonin regulating phosphate excretion, but FGF23 may work as a "memory hormone" and a long-term regulator of a positive phosphate balance. This behaviour may be more comparable to the marker protein of diabetes control, HbA1C, with the additional property that FGF23 not just represents, but also acts on phosphate control. Thus, these insights suggest the existence of additional acute regulatory factors of phosphate balance which remain to be identified in the future.

\section{Treatment of Phosphate Retention in Predialysis CKD: Diets}

Therapeutic approaches aiming at phosphate lowering (i.e., serum levels as well as phosphate load) include reducing oral intake of phosphate by dietary modifications as well as intestinal absorption using phosphate binders. Phosphaterestricted diets should be designed in a manner that avoids simultaneous protein malnutrition. In this context, there are a couple of studies supporting that at least moderate phosphate restriction is well tolerated in most individuals. The use of phosphate-restricted diets in combination with oral phosphate binders has become well established in the management of patients with CKD Stages 3-5CKD (including CKD Stage 5D). Recent epidemiological studies in larger cohorts of dialysis patients are uniequivocal regarding associations between dietary phosphate restriction and survival benefits $[13,14]$.

Two prospective studies are available specifically evaluating the impact of phosphate-restricted diets in CKD patients. Zeller et al. showed that the restriction of dietary protein plus phosphate intake was welltolerated and maintained nutritional parameters in a satisfying range in a study of 35 Type I diabetes patients with associated nephropathy [15]. Here, a significant reduction in urinary phosphate excretion in the group assigned to a protein/phosphate restriction as compared to subjects receiving a control diet was found. There was no evaluation of other CKD-MBD-related parameters performed in this investigation. Lafage-Proust and colleagues reported in another study featuring a very strict low-protein, low-phosphorus diet (supplemented with essential amino acids and their ketoanalogs) that after a follow-up of five years bone formation rate was normal or high in 10 patients, and low in the remaining 6, without observing low-proteinassociated malnutrition in any of the participants [16].

A new view on phosphate restriction currently develops based on considerations that there might be a significant difference between naturally occuring phosphate and phosphate supplements in food [17-19]. Natural phosphate is 
TABLE 1: KDIGO clinical practice guideline for the diagnosis, evaluation, prevention, and treatment of Chronic Kidney Disease-Mineral and Bone Disorder (CKD-MBD) [14]: recommendations for the management of phosphate in predialysis patients.

(4.1.1) In patients with CKD stages 3-5, we suggest maintaining serum phosphorus in the normal range (2C). In patients with CKD stages 3-5 (2D) and 5D (2B), we suggest using phosphate binding agents in the treatment of

(4.1.4) hyperphosphatemia. It is reasonable that the choice of phosphate binder takes into account CKD stage, presence of other components of CKD-MBD, concomitant therapies, and side effect profile (not graded).

(4.1.5) In patients with CKD stage 3-5D and hyperphosphatemia, we recommend restricting the dose of calcium-based phosphate binder and/or the dose of calcitriol or vitamin D analog in the presence of persistent or recurrent hypercalcemia (1B).

In patients with CKD stage 3-5D and hyperphosphatemia, we suggest restricting the dose of calcium-based phosphate binders in the presence of arterial calcification (2C) and/or adynamic bone disease (2C) and/or if serum PTH levels are persistently low (2C).

(4.1.6) In patients with CKD stage 3-5D we recommend avoiding long term use of aluminum containing phosphate binders and in patients with $\mathrm{CKD}$ stage $5 \mathrm{D}$ avoiding dialysate aluminum contamination to prevent aluminum intoxication (1C).

In patients with CKD stages $3-5 \mathrm{D}$, we suggest
in combination with other treatments $(2 D)$.

mostly contained in food as organic esters or as phytates (hexa-phospho-inosites), and this bound phosphate is only absorbed at a rate between $40-60 \%$ at maximum [20-22]. In contrast, phosphate-containing food additives include polyphosphates, diphosphates, triphosphates, potassium phosphate, calcium phosphate and so on, while this "free" phosphate is quite readily absorbed [19]. Recently, it became evident from longitudinal observations of the "Chronic Renal Insufficiency Cohort" (CRIC) cohort that patients with mild to moderate renal insufficiency had a $2.5-2.7$-fold increased risk of hyperphosphatemia $(>4,6 \mathrm{mg} / \mathrm{dL}$ ) when the tlowest income tertile was compared to the highest income tertile, despite equal amounts of phosphate intake $(1,156$ versus $1,190 \mathrm{mg} / \mathrm{Tag}$ ) [23]. This difference could only be explained by differences in "phosphate quality," with a higher intake of the so-called "fast food" (which is particularly rich in phosphate additives) in the lower income group.

\section{Treatment of Phosphate Retention in Predialysis CKD: Phosphate Binders}

Phosphate binders are pragmatically used in predialysis patients when hyperphosphatemia becomes apparent however, thresholds for treatment initiation vary a lot and are mostly based on preferences at the discretion of the individual physician. The KDIGO guidelines 2009 (Table 1) suggest to maintain phosphate serum levels within the normal range in CKD patients in stages 3-5 [24], but mild hyperphosphatemia can still be considered an underrecognized and underestimated phenomenon in predialysis patients. Calcium-containing binders may globally play a predominant role as the first-line choice of phosphate binder therapy in these predialysis stages, because they are relatively inexpensive and because these patients tend to have calcium serum levels in the lower or even below the normal range.

Since many CKD patients suffer from a low mineral density, there is also the question, whether a negative calcium balance must be avoided anyway, but there is no answer on the harmless or even beneficial calcium intake quantities that can be permitted. However, we also know quite well that increased calcium exposure, or a positive net calcium balance, may predispose $C K D$ patients for the risk of developing cardiovascular calcification $[25,26]$.

Of note, with the exception of calcium carbonate in many European countries, phosphate binder treatment had generally been an off-label use in these CKD stages tolerated by most authorities until recently. In 2009, however, sevelamer carbonate was approved by the EMA in the indication of treating CKD patients not on dialysis with phosphate serum levels of above $1.78 \mathrm{mmol} / \mathrm{L}$ in Europe. The same label was also approved for lanthanum carbonate a few months later.

There are only few publications available on phosphate binder use in predialysis patients. Such studies are furthers hampered by the fact that phosphate-lowering effects are not clearly visible, because a decline in phosphaturia may blunt the changes in circulating phosphate levels.

The efficacy of sevelamer carbonate in predialysis CKD patients was recently examined in 19 nephrology centers across Northern Europe and Australia in a single-arm study approach with a poststudy washout period [27]. Investigators initially screened 129 patients, 41 of whom completed the study. Recruitment was heavily influenced by screening failure, because the cutoff of phosphate serum levels was set very high $(5.5 \mathrm{mg} / \mathrm{dL})$, according to authority requirements in the study protocol, which also caused a high inclusion percentage of patients in CKD stage 5. Sevelamer carbonate lowered serum phosphorus levels by a mean of $1.4 \pm \mathrm{SD}$ $1.0 \mathrm{mg} / \mathrm{dL}(P<.001)$ during an eight-week observation period. $70 \%$ of those patients with stage 4 CKD achieved normal serum levels of $2.7-4.6 \mathrm{mg} / \mathrm{dL}$. Serum bicarbonate concentrations rose significantly from $16.6 \pm 3.6 \mathrm{mEq} / \mathrm{L}$ to $18.2 \pm 3.7 \mathrm{mEq} / \mathrm{L}(P=.005)$ which may be clinically meaningful given the high prevalence of metabolic acidosis in CKD stage 4-5 patients. The mean prescribed dosage after dosage titration was $7.8 \mathrm{~g} /$ day sevelamer carbonate at the end of the study.

Lanthanum carbonate was also studied recently in a Phase II trial in CKD stage 3 and 4 patients [28]. 121 hyperphosphatemic patients were randomized in a $2: 1$ manner to lanthanum or placebo, respectively. $44.6 \%$ of lanthanumtreated patients reached normophosphatemia versus $26.5 \%$ 
in the placebo group, with safety profile and tolerability on a strictly comparable level. The mean dose of lanthanum carbonate after eight weeks was 2,645 $\pm 97 \mathrm{mg} /$ day.

The RIND study provided some more indirect insights into the clinical potential of phosphate binder treatment in predialysis stages [25]. This investigation was designed as a prospective trial observing the progression of coronary artery calcifications in incident hemodialysis patients treated with either sevelamer-HCl or calcium acetate. One central observation was that even though none of the patients who were free of calcifications became positive during the first 18 months of dialysis treatment, "progressors" (about 60\% of the total cohort) were all calcified prior to initiation of dialysis treatment. Thus, prevention strategies targeting cardiovascular calcification, such as phosphate-lowering approaches, must be initiated early in the course of CKD.

Currently, there is only one prospective clinical study addressing the biological effect of phosphate binders in CKD patients [29]. Russo and colleagues studied 90 phosphate binder-naive patients in predialysis CKD stages 3-5 with regard to progression of coronary artery calcification. Patients were randomized ( 30 per group) to either a low $\mathrm{P}$ diet alone, or to a low $\mathrm{P}$ diet in combination with fixed doses of calcium carbonate ( $2 \mathrm{~g} /$ day $)$ or sevelamer ( $1.6 \mathrm{~g} /$ day). Final MSCT-based coronary artery calcification scores were greater than initial scores in those subjects receiving diet and diet in combination with calcium carbonate, while no progression was observed in the diet plus sevelamer-treated group. Absolute urinary phosphate excretion was higher in the diettreated patients but lower when compared to baseline in those receiving phosphate binders. FGF23 serum levels were unfortunately not available from this study.

\section{Novel Concepts for Phosphate Management in Predialysis CKD Stages}

One of the key issues in phosphate management in predialysis stages of CKD is the fact that serum phosphate levels do not appropriately reflect phosphate retention. Because phosphate retention is immediately counterregulated by phosphatonins such as FGF23, PTH, and probably other factors, composite measurements of these parameters may need to find their way into diagnostic algorithms, as well as estimates of phosphate excretion in the urine. Nagano et al. recently demonstrated in a uremic rat model that phosphate binding by sevelamer dose-dependently lowered both FGF23 and PTH serum concentrations [30]. In this line, two clinical pilot studies were launched investigating the effect of phosphate binder treatment on these surrogate parameters of phosphate retention. Isakova et al. studied 16 normophosphatemic CKD stage 3 and 4 patients to either lanthanum carbonate or placebo, and to either a $750 \mathrm{mg}$ or $1,500 \mathrm{mg}$ phosphate diet, in a $2 \times 2$ factorial design [31]. FGF23, PTH, serum levels of calcium and phosphate, and phosphate excretion were measured. While the high phosphate diet and lanthanum treatment lead to decreases in phosphate excretion, no effects were observed with regard to FGF23 and PTH levels, respectively. However, the high phosphate diet caused a significant increase in FGF23 levels.
Block and colleagues started a similar, but larger pilot study in >200 normophosphatemic CKD patients (eGFR 20-45 $\mathrm{ml} / \mathrm{min}$ ) using three different phosphate binders (lanthanum carbonate, sevelamer carbonate, calcium acetate) with matching placebos [32]. Follow-up will be nine months, and parameters of interest are serum concentrations of FGF23, PTH, calcium, and phosphate, as well as changes in arterial stiffness (pulse wave velocity) and coronary artery celacification scores (multislice spiral CT). Data are expected to be available in autumn 2011.

The biologically plausible hypothesis that "invisible" phosphate retention may be the earliest and foremost event in the development of secondary hyperparathyroidism was already picked up in concept papers [33]. Here, a "phosphocentric" (in contrast a "vitamin D-centric") view was proposed with the consequence that the initial treatment approach must be targeted towards limiting phosphate ingestion and absorption in predialysis patients. This view was recently supported by Evenepoel et al. who demonstrated that urinary phosphate excretion in predialysis CKD patients was indeed highest in individuals with both elevated FGF23 and PTH, respectively, and that increased FGF23 and phosphate, and decreased 25-OH-vitamin D were independently associated with decreased calcitriol levels [34]. What needs to be determined and better understood though is, whether, or to which degree, secondary hyperparathyroidism (and FGF23 elevations) is a favourable, adaptive or a disadvantageous, maladaptive mechanism, prior to basing treatment decisions on this novel concept. Furthermore, age, klotho expression, and potentially klotho resistance (in uremia) may impact on shifting phosphate regulation to dysregulation $[3,9,10,12]$.

\section{Conclusion}

Much needs to be learned about the assessment and the impact of deranged phosphate homeostasis in predialysis CKD stages. Limited data suggest that phosphate retention may cause harm in these stages even if serum levels do not rise beyond the normal range. Composite measures of serum phosphate, fractional phosphate excretion, and FGF23 may become an integral and improved approach in therapeutic decision making, in contrast to just waiting for hyperphosphatemia to occur. However, there is a huge gap with regard to prospective clinical trials assessing the effect of phosphatelowering strategies on clinically meaningful hard endpoints. In addition, since a positive phosphate balance may already tip the balance towards cardiovascular calcification and damage, we require information on the impact of treatment on cardiovascular surrogate parameters as well. Fortunately, there are initiatives underway attempting to at least partially fill those gaps of knowledge.

\section{References}

[1] T. Isakova, O. Gutierrez, A. Shah et al., "Postprandial mineral metabolism and secondary hyperparathyroidism in early CKD," Journal of the American Society of Nephrology, vol. 19, no. 3, pp. 615-623, 2008. 
[2] A. A. Portale, B. P. Halloran, and R. C. Morris, "Dietary intake of phosphorus modulates the circadian rhythm in serum concentration of phosphorus. Implications for the renal production of 1,25-dihydroxyvitamin D," The Journal of Clinical Investigation, vol. 80, no. 4, pp. 1147-1154, 1987.

[3] T. Berndt and R. Kumar, "Novel mechanisms in the regulation of phosphorus homeostasis," Physiology, vol. 24, no. 1, pp. 17 25, 2009.

[4] Y. Nishida, Y. Taketani, H. Yamanaka-Okumura et al., "Acute effect of oral phosphate loading on serum fibroblast growth factor 23 levels in healthy men," Kidney International, vol. 70, no. 12, pp. 2141-2147, 2006.

[5] B. Kestenbaum, J. N. Sampson, K. D. Rudser et al., "Serum phosphate levels and mortality risk among people with chronic kidney disease," Journal of the American Society of $\mathrm{Ne}$ phrology, vol. 16, no. 2, pp. 520-528, 2005.

[6] C. Y. Hsu and G. M. Chertow, "Elevations of serum phosphorus and potassium in mild to moderate chronic renal insufficiency," Nephrology Dialysis Transplantation, vol. 17, no. 8, pp. 1419-1425, 2002.

[7] A. Levin, G. L. Bakris, M. Molitch et al., "Prevalence of abnormal serum vitamin $\mathrm{D}, \mathrm{PTH}$, calcium, and phosphorus in patients with chronic kidney disease: results of the study to evaluate early kidney disease," Kidney International, vol. 71, no. 1, pp. 31-38, 2007.

[8] O. Gutierrez, T. Isakova, E. Rhee et al., "Fibroblast growth factor-23 mitigates hyperphosphatemia but accentuates calcitriol deficiency in chronic kidney disease," Journal of the American Society of Nephrology, vol. 16, no. 7, pp. 2205-2215, 2005.

[9] S. Liu and L. D. Quarles, "How fibroblast growth factor 23 works," Journal of the American Society of Nephrology, vol. 18, no. 6, pp. 1637-1647, 2007.

[10] D. Prié, P. U. Torres, and G. Friedlander, "Latest findings in phosphate homeostasis," Kidney International, vol. 75, no. 9, pp. 882-889, 2009.

[11] M. Wolf, "Forging forward with 10 burning questions on FGF23 in kidney disease," Journal of the American Society of Nephrology, vol. 21, no. 9, pp. 1427-1435, 2010.

[12] J. Silver and T. Naveh-Many, "Phosphate and the parathyroid," Kidney International, vol. 75, no. 9, pp. 898-905, 2009.

[13] N. Noori, K. Kalantar-Zadeh, C. P. Kovesdy, R. Bross, D. Benner, and J. D. Kopple, "Association of dietary phosphorus intake and phosphorus to protein ratio with mortality in hemodialysis patients," Clinical Journal of the American Society of Nephrology, vol. 5, no. 4, pp. 683-692, 2010.

[14] K. E. Lynch, R. Lynch, G. C. Curhan, and S. M. Brunelli, "Prescribed dietary phosphate restriction and survival among hemodialysis patients," Clinical Journal of the American Society of Nephrology, vol. 6, no. 3, pp. 620-629, 2011.

[15] K. Zeller, E. Whittaker, L. Sullivan, P. Raskin, and H. R. Jacobson, "Effect of restricting dietary protein on the progression of renal failure in patients with insulin-dependent diabetes mellitus," The New England Journal of Medicine, vol. 324, no. 2, pp. 78-84, 1991.

[16] M. H. Lafage-Proust, C. Combe, N. Barthe, and M. Aparicio, "Bone mass and dynamic parathyroid function according to bone histology in nondialyzed uremic patients after longterm protein and phosphorus restriction," Journal of Clinical Endocrinology and Metabolism, vol. 84, no. 2, pp. 512-519, 1999.

[17] C. Sullivan, S. S. Sayre, J. B. Leon et al., "Effect of food additives on hyperphosphatemia among patients with end-stage renal disease: a randomized controlled trial," JAMA, vol. 301, no. 6, pp. 629-635, 2009.

[18] J. Uribarri, "Phosphorus homeostasis in normal health and in chronic kidney disease patients with special emphasis on dietary phosphorus intake," Seminars in Dialysis, vol. 20, no. 4, pp. 295-301, 2007.

[19] K. Kalantar-Zadeh, L. Gutekunst, R. Mehrotra et al., "Understanding sources of dietary phosphorus in the treatment of patients with chronic kidney disease," Clinical Journal of the American Society of Nephrology, vol. 5, no. 3, pp. 519-530, 2010.

[20] L. H. Kayne, D. Z. D’Argenio, J. H. Meyer, M. S, Hu, N. Jamgotchian, and D. B. N. Lee, "Analysis of segmental phosphate absorption in intact rats. A compartmental analysis approach," The Journal of Clinical Investigation, vol. 91, no. 3, pp. 915-922, 1993.

[21] L. Bohn, A. S. Meyer, and S. K. Rasmussen, "Phytate: impact on environment and human nutrition. A challenge for molecular breeding," Journal of Zhejiang University: Science B, vol. 9, no. 3, pp. 165-191, 2008.

[22] X. G. Lei and J. M. Porres, "Phytase enzymology, applications, and biotechnology," Biotechnology Letters, vol. 25, no. 21, pp. 1787-1794, 2003.

[23] O. M. Gutiérrez, C. Anderson, T. Isakova et al., "Low socioeconomic status associates with higher serum phosphate irrespective of race," Journal of the American Society of Nephrology, vol. 21, no. 11, pp. 1953-1960, 2010.

[24] "Kidney disease: improving Global Outcomes (KDIGO) CKD-MBD Work Group. KDIGO clinical practice guideline for the diagnosis, evaluation, prevention, and treatment of Chronic Kidney Disease-Mineral and Bone Disorder (CKDMBD)," Kidney International. Supplement, no. 113, pp. S1S130, 2009.

[25] G. A. Block, D. M. Spiegel, J. Ehrlich et al., "Effects of sevelamer and calcium on coronary artery calcification in patients new to hemodialysis," Kidney International, vol. 68, no. 4, pp. 1815-1824, 2005.

[26] W. G. Goodman, J. Goldin, B. D. Kuizon et al., "Coronaryartery calcification in young adults with end-stage renal disease who are undergoing dialysis," The New England Journal of Medicine, vol. 342, no. 20, pp. 1478-1483, 2000.

[27] M. Ketteler, M. Rix, S. Fan et al., "Efficacy and tolerability of sevelamer carbonate in hyperphosphatemic patients who have chronic kidney disease and are not on dialysis," Clinical Journal of the American Society of Nephrology, vol. 3, no. 4, pp. 11251130, 2008.

[28] S. M. Sprague, H. Abboud, P. Qiu, M. Dauphin, P. Zhang, and W. Finn, "Lanthanum carbonate reduces phosphorus burden in patients with CKD stages 3 and 4: a randomized trial," Clinical Journal of the American Society of Nephrology, vol. 4, no. 1, pp. 178-185, 2009.

[29] D. Russo, I. Miranda, C. Ruocco et al., "The progression of coronary artery calcification in predialysis patients on calcium carbonate or sevelamer," Kidney International, vol. 72, no. 10, pp. 1255-1261, 2007.

[30] N. Nagano, S. Miyata, M. Abe et al., "Effect of manipulating serum phosphorus with phosphate binder on circulating PTH and FGF23 in renal failure rats," Kidney International, vol. 69, no. 3, pp. 531-537, 2006.

[31] T. Isakova, O. M. Gutirrez, K. Smith et al., "Pilot study of dietary phosphorus restriction and phosphorus binders to target fibroblast growth factor 23 in patients with chronic kidney disease," Nephrology Dialysis Transplantation, vol. 26, no. 2, pp. 584-591, 2011. 
[32] G. A. Block, M. S. Persky, M. Ketteler et al., "A randomized double-blind pilot study of serum phosphorus normalization in chronic kidney disease: a new paradigm for clinical outcomes studies in nephrology," Hemodialysis International, vol. 13 , no. 3, pp. 360-362, 2009.

[33] J. B. Wetmore and L. D. Quarles, "Calcimimetics or vitamin $\mathrm{D}$ analogs for suppressing parathyroid hormone in end-stage renal disease: time for a paradigm shift?" Nature Clinical Practice Nephrology, vol. 5, no. 1, pp. 24-33, 2009.

[34] P. Evenepoel, B. Meijers, L. Viaene et al., "Fibroblast growth factor-23 in early chronic kidney disease: additional support in favor of a phosphate-centric paradigm for the pathogenesis of secondary hyperparathyroidism," Clinical Journal of the American Society of Nephrology, vol. 5, no. 7, pp. 1268-1276, 2010. 


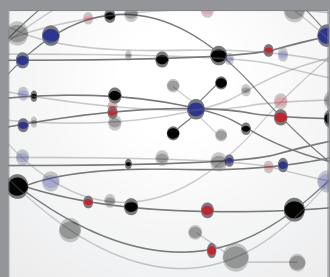

The Scientific World Journal
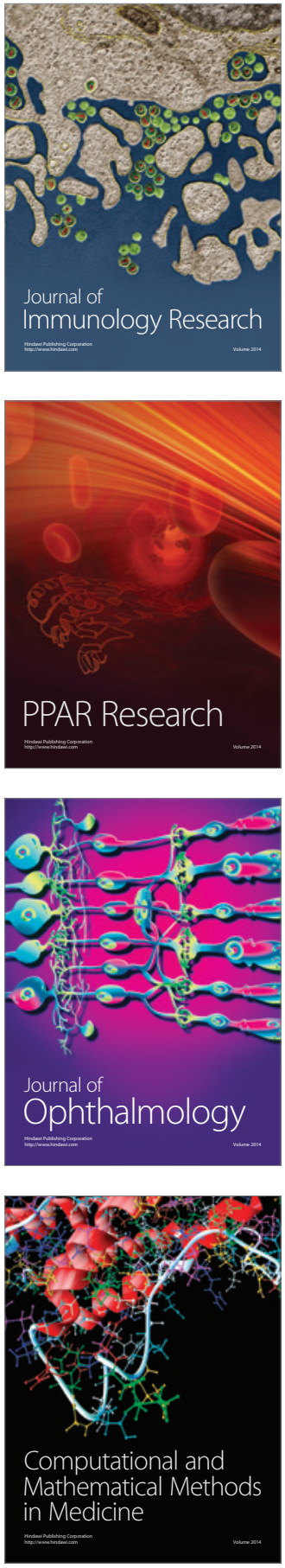

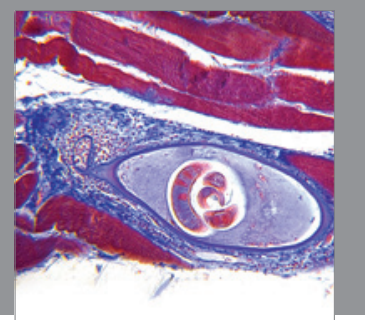

Gastroenterology

Research and Practice
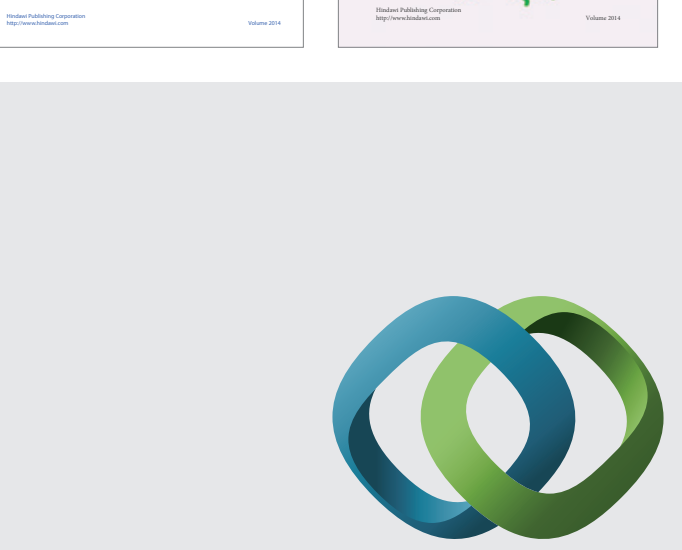

\section{Hindawi}

Submit your manuscripts at

http://www.hindawi.com
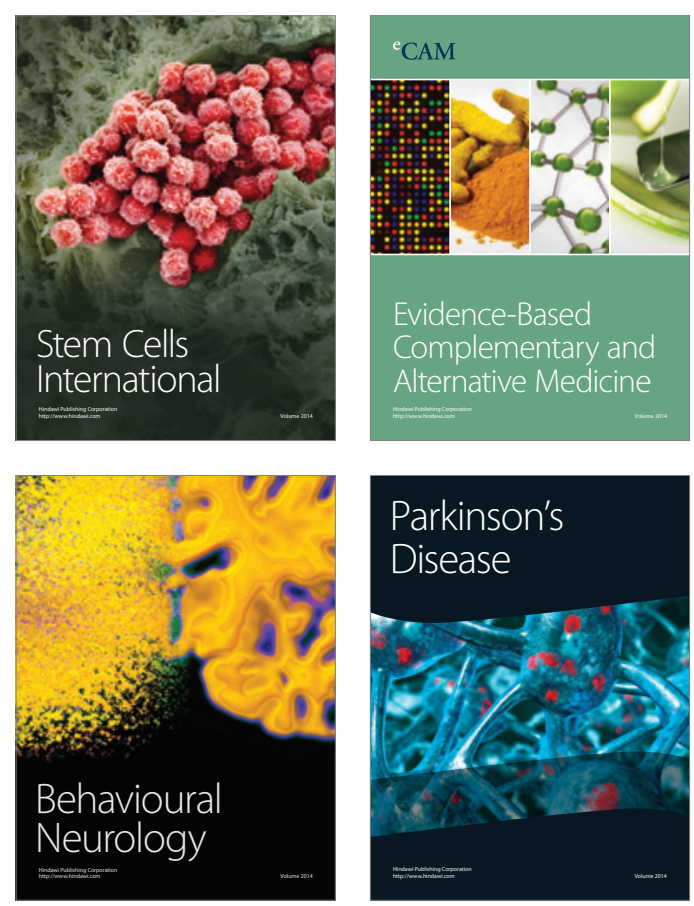

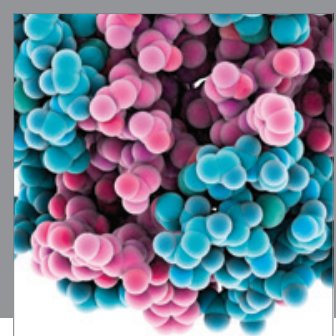

Journal of
Diabetes Research

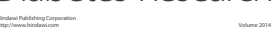



Disease Markers
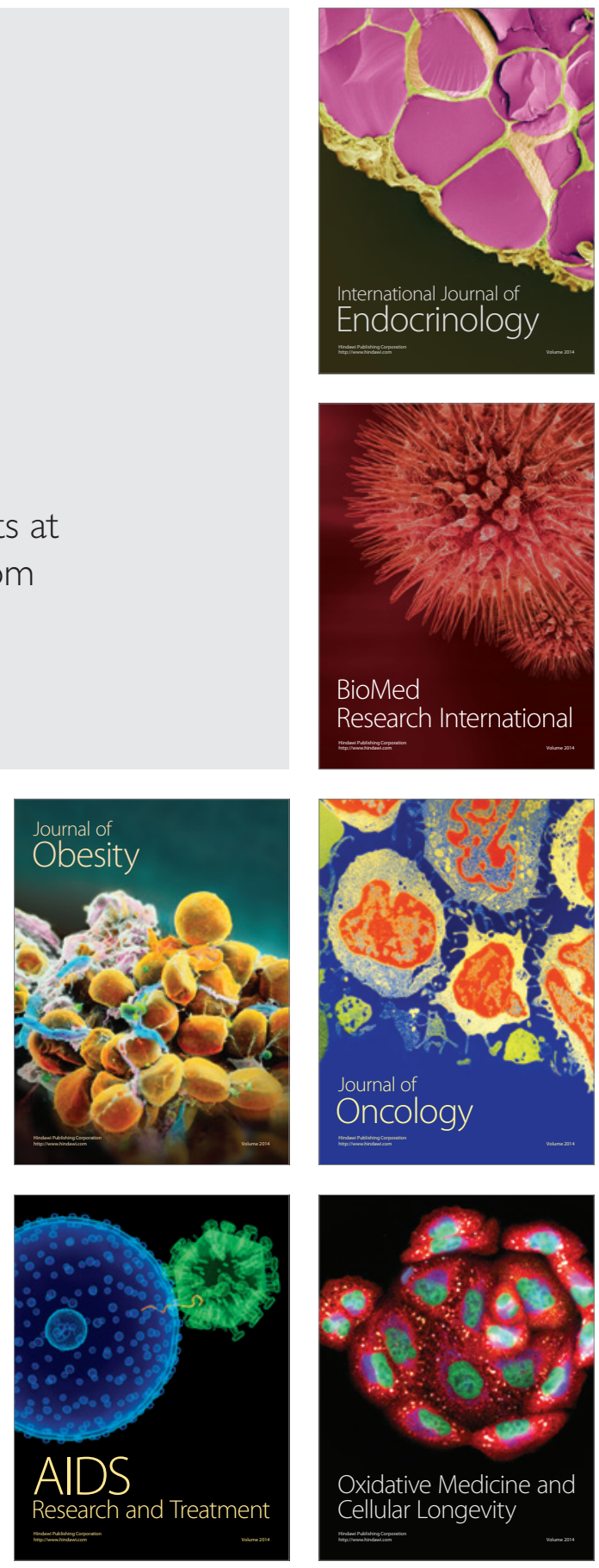